\title{
Creating Inclusive, Supportive, and Safe Environments in Planetary Science for Members of the LGBTQ+ Community
}

\author{
A White Paper in support of the 2023-2032 Planetary Science and Astrobiology Decadal \\ Survey
}

Lead Author: Kathleen E. Vander Kaaden, Phone: 281-244-5020, Affiliation: Jacobs, JETS

Contract, NASA Johnson Space Center (JSC), Kathleen.e.vanderkaaden@nasa.gov

Co-Authors: Catheryn Ryan (University of Western Ontario), Edgard G. Rivera-Valentín (Lunar and Planetary Institute (USRA) - LPI), Cynthia B. Phillips (Jet Propulsion Laboratory), James Haber (Purdue University), Justin Filiberto (LPI, USRA), Adeene Denton (Purdue University)

\section{Endorsers:}

Paul Abell ${ }^{19}$, Brendan A. Anzures ${ }^{4}$, Rosalind Armytage ${ }^{9}$, Jodi Berdis ${ }^{20}$, Kristin M. Block ${ }^{37}$, Schuyler Borges ${ }^{23}$, Ali M. Bramson $^{27}$, Michael W. Busch ${ }^{30}$, Paul K. Byrne ${ }^{22}$, CAPTEM, Nancy Chabot ${ }^{11}$, Barbara Cohen ${ }^{3}$, Kerri Donaldson Hanna $^{39}$, Stephen Elardo ${ }^{40}$, Michael E. Evans ${ }^{19}$, Katelyn R. Frizzell ${ }^{28}$, Martha Gilmore ${ }^{50}$, Timothy A. Goudge ${ }^{34}$, Jennifer (JA) Grier ${ }^{26}$, Juliane Gross ${ }^{28}$, Jennifer Hanley ${ }^{14}$, Romy Hanna ${ }^{10}$, Tanya Harrison ${ }^{25}$, Adam Leah W. Harvey ${ }^{46}$, Steven A. Hauck, II ${ }^{6}$, Joseph Razzell Hollis ${ }^{5}$, Timothy R Holt ${ }^{48}$, Briony Horgan ${ }^{27}$, Sarah Horst ${ }^{12}$, Samuel M. Howell ${ }^{5}$, Kayla Iacovino ${ }^{9}$, James T. Keane ${ }^{5}$, Michelle R. Kirchoff ${ }^{31}$, Rachel Klima ${ }^{11}$, Christian Klimczak ${ }^{41}$, Joshua Knicely ${ }^{35}$, Dara Laczniak ${ }^{27}$, Michaela Leung ${ }^{38}$, Timothy A. Livengood ${ }^{43}$, Nicole Lunning ${ }^{19}$, Angela G Marusiak ${ }^{5}$, Marisa Mayer $^{32}$, Maggie McAdam ${ }^{16}$, Ryan McCabe ${ }^{7}$, Francis M. McCubbin ${ }^{19}$, Claire McLeod ${ }^{15}$, Moses Milazzo ${ }^{24}$, Julie L Mitchell $^{19}$, Jamie L. Molaro ${ }^{26}$, Gordon Moore ${ }^{9}$, Sarah E. Moran ${ }^{12}$, Catherine Neish ${ }^{26}$, Claire E. Newman ${ }^{1}$, Jessica Noviello $^{29}$, Charlotte Olsen ${ }^{28}$, Amanda Ostwald ${ }^{47}$, Kellye M Pando ${ }^{9}$, Anne Peslier ${ }^{9}$, Erika Rader ${ }^{42}$, Zia Rahman ${ }^{9}$, Elizabeth Rampe ${ }^{19}$, Julie Rathbun ${ }^{26}$, Soumya Ray ${ }^{2}$, Darryl Reano ${ }^{33}$, Aaron B. Regberg ${ }^{19}$, Antonio J. Ricco ${ }^{17}$, Jacob Richardson $^{45}$, Christina Richey ${ }^{5}$, Stuart J. Robbins ${ }^{31}$, James H. Roberts ${ }^{11}$, Emilie Royer ${ }^{26}$, Amanda Rudolph ${ }^{27}$, Alicia Rutledge $^{23}$, Andrew Ryan ${ }^{36}$, SBAG Steering Committee, Noel A. Scudder ${ }^{27}$, Jennifer Scully ${ }^{5}$, Alexander Sehlke ${ }^{18}$, Rachel Sheppard ${ }^{5}$, Linda Spilker ${ }^{5}$, Sabine Stanley ${ }^{12}$, Beck E. Strauss ${ }^{21}$, Marshall J. Styczinski ${ }^{49}$, Michelle Thompson ${ }^{27}$, Valerie M. Tu ${ }^{9}$, Arya Udry ${ }^{47}$, Sarah Valencia ${ }^{45}$, Steven Vance ${ }^{5}$, Dany Waller ${ }^{12}$, Nicole Whelley ${ }^{44}$, Hayley N. Williamson $^{8}$, Marcella Yant ${ }^{13}$

\footnotetext{
${ }^{1}$ Aeolis Research, Chandler, AZ, ${ }^{2}$ Arizona State University, ${ }^{3}$ Barbara A. Cohen, ${ }^{4}$ Brown University, ${ }^{5}$ Jet Propulsion Laboratory, Californian Institute of Technology, ${ }^{6}$ Case Western Reserve University, ${ }^{7}$ Hampton University, ${ }^{8}$ IRF, ${ }^{9} \mathrm{Jacobs},{ }^{10} \mathrm{Jackson}$ School of Geosciences, University of Texas at Austin, ${ }^{11} \mathrm{Johns}$ Hopkins University Applied Physics Laboratory, ${ }^{12}$ Johns Hopkins University, ${ }^{13}$ Lockheed Martin, ${ }^{14}$ Lowell Observatory, ${ }^{15}$ Miami University, OH, ${ }^{16}$ NASA Ames Research Center, ${ }^{17}$ NASA Ames Research Center / Stanford University, ${ }^{18}$ NASA Ames Research Center/ Bay Area Environmental Research Institute, ${ }^{19}$ NASA Johnson Space Center, ${ }^{20}$ New Mexico State University, ${ }^{21}$ NIST \& NASA GSFC, ${ }^{22}$ North Carolina State University, ${ }^{23}$ Northern Arizona University, ${ }^{24}$ Other Orb LLC, ${ }^{25}$ Planet Labs, ${ }^{26}$ Planetary Science Institute, ${ }^{27}$ Purdue University, ${ }^{28}$ Rutgers University, ${ }^{29}$ School of Earth and Space Exploration, Arizona State University, ${ }^{30}$ SETI Institute, ${ }^{31}$ Southwest Research Institute, ${ }^{32}$ Stanford University, ${ }^{33}$ STEM Transformation Institute, Florida International University, ${ }^{34}$ The University of Texas at Austin, ${ }^{35}$ University of Alaska Fairbanks, ${ }^{36}$ University of Arizona, ${ }^{37}$ University of Arizona/Lunar and Planetary Laboratory, ${ }^{38}$ University of California, Riverside, ${ }^{39}$ University of Central Florida, ${ }^{40}$ University of Florida, ${ }^{41}$ University of Georgia, ${ }^{42}$ University of Idaho, ${ }^{43}$ University of Maryland, ${ }^{44}$ University of Maryland - College Park / NASA GSFC, ${ }^{45}$ University of Maryland, College Park, ${ }^{46}$ University of Maryland, Baltimore County, ${ }^{47}$ University of Nevada, Las Vegas, ${ }^{48}$ University of Southern Queensland, ${ }^{49}$ University of Washington, ${ }^{50}$ Wesleyan University
} 


\subsection{INTRODUCTION}

"I believe that no one should ever have to choose between a career we love and living our lives with authenticity and integrity." These words, spoken by Out and Equal Executive Director Selisse Berry, express the criticality of fostering an interdisciplinary, diverse, equitable, inclusive, and accessible environment over the next decade in the field of planetary science, especially for members of the LGBTQ+ community. Prior to discussing the issues faced by the LGBTQ+ community in planetary science and our recommendations to mitigate these concerns, we note that members of the community utilize various acronyms to describe a person's sexual orientation or gender identity, but for the purposes of this paper, we will use the acronym LGBTQ+ to include any and all members of this marginalized group. LGBTQ+ stands for Lesbian, Gay, Bisexual, Transgender, Queer, and the "+" signifies the importance that one acronym cannot possibly capture everyone's experience of their gender identity, expression, and/or sexual orientation. In addition, although this paper speaks specifically to creating inclusive, supportive, and safe environments in planetary science for members of the LGBTQ+ community, we strongly encourage the decadal survey to consider the state of the profession and the issues of equity, diversity, inclusion, and accessibility - not as separable issues, but as critical, interrelated steps on the pathway to understanding the complex nature of our solar system and beyond.

A 2020 survey of the planetary sciences workforce indicates that LGBTQ+ representation has grown in planetary science from 3\% in 1970 to $12 \%$ in the last 5 years (see Section 2.0), yet many members of this community are marginalized, excluded, and unsafe in various aspects of this field (e.g., Ackerman et al., 2019). In fact, a survey of LGBTQ scientists in the UK showed that almost a third of the participants considered leaving the physical sciences due to negative workplace experiences (Gibney, 2019; Marín-Spiotta, 2020). Cech and Pharm (2017) showed that "LGBT employees in STEM agencies report systematically more negative workplace experiences than their non-LGBT colleagues". Similarly, Richey et al. (2019) showed that LGBTQ+ "women and gender minorities experience a more hostile workplace climate in astronomy and planetary science compared to cisgender, straight women". Studies of this nature have brought the negative experiences of LGBTQ+ scientists to light, and in doing so, provide us the catalyst to make meaningful, lasting changes in our community to create inclusive, supportive, and safe environments where they can thrive over the next decade.

A recent study by Mattheis et al. (2019) found that "heteronormative assumptions frequently silence conversations about gender and sexuality in STEM workplaces and result in complicated negotiations of self for queer professionals". These assumptions and added burden can have detrimental impacts on the LGBTQ+ community, including feelings of isolation, exclusion, and decreased work satisfaction resulting in poor performance or leaving the field all together. These issues are compounded for members of the LGBTQ+ community that also identify with other underrepresented groups such as BIPOC (Black, Indigenous, and People of Color) and/or people with disabilities (see decadal white papers by Diniega et al. and Piatek et al.). These white papers should be consulted to ensure members falling into multiple marginalized groups are adequately supported. In preparing for the next decade of science through the decadal survey, we have the opportunity to change the environment in which we learn and work to be more inclusive and diverse, while fostering a culture of caring to ensure the safety of all of our community members.

The aim of this white paper is to provide recommendations to NASA and colleagues within

the field of planetary science on how to work towards making our field accessible to members of the LGBTQ+ community by creating an inclusive, supportive, and safe environment to facilitate their success. 


\subsection{DEMOGRAPHICS}

The American Astronomical Society's (AAS) Division of Planetary Sciences (DPS) 2020 workforce survey (Hendrix et al., 2020) included questions about sexual orientation and gender expression and identity. Respondents included student and non-student researchers. Overall, $12.6 \%$ of respondents identified as lesbian/gay/bisexual; using a margin of error with a $95 \%$ confidence, one would expect that $12.6 \% \pm 1.5 \%$ of the planetary science field would identify as LGB. Specifically, regarding sexual orientation, $2.2 \%$ of respondents identified as other, $2.9 \%$ as lesbian or gay, $6.3 \%$ as bisexual, $78.8 \%$ as heterosexual, and $9.9 \%$ of respondents preferred not to answer. With regards to transgender identity, $0.8 \%$ of respondents identified as transgender, $96.6 \%$ as not, and $2.6 \%$ preferred not to answer. With regards to gender, $1.1 \%$ identified as nonbinary or other identity, $36.1 \%$ as women, $60 \%$ as men, and $2.7 \%$ of respondents preferred not to answer. For more information on nonbinary gender inclusivity, see the decadal white paper by Strauss et al. submitted to this same call.

Results from the demographic survey also indicated that LGBTQ+ representation has significantly grown over time. Cross-correlation of answers to questions regarding LGBTQ+ identity and terminal degree conferral date indicates that LGBTQ+ representation has grown in planetary science from $3 \%$ in 1970 to $12 \%$ in the last 5 years, suggesting a growth rate of $0.2 \%$ per year. Such growth is also apparent when comparing survey responses from non-student and student researchers, of which $0.7 \%$ and $2.6 \%$ are gender nonbinary, respectively. Additionally, cross-correlating answers regarding LGBTQ+ identity and job type (i.e., tenured, tenure-track, hard money, soft money, and postdoctoral), indicated that on average LGBTQ+ respondents are represented at $11.3 \%$ in postdoctoral and tenure-track positions, while they are represented at $4.8 \%$ in tenured positions, and $6.9 \%$ in all other positions. Thus, these demographics suggest that the planetary science LGBTQ+ community is represented at or above the national average. Indeed, Newport $(2018)^{1}$ showed that $4.5 \%$ of the U.S. population identified as LGBT. Although no national statistics exist for nonbinary gender identity, a recent membership survey by the Geological Society of America ${ }^{2}$ (GSA) found that $\sim 0.1 \%$ of respondents identified as nonbinary; an order of magnitude less than in planetary science.

Although the demographic data indicates that in planetary science LGBTQ+ scientists are not underrepresented, unlike some axes of representation (see decadal white paper by Rivera-Valentín et al. for more information), such a diversity study is only half of the discussion. Diversity and inclusion initiatives require the consideration of representation and visibility, as well as workforce issues, such as harassment, bullying, and unequal treatment in the workplace. Indeed, a recent survey found that LGBTQ+ planetary scientists experienced more verbal harassment and were twice more likely to be physically harassed due to their LGBTQ+ identity (Richey et al., 2019). Until a recent Supreme Court ruling (case no. 17-1618 ${ }^{3}$ ), LGBTQ+ status, unlike race and gender, was not a protected status in the U.S. In fact, until summer 2020 in 28 of 50 states, it was legal to fire someone because they were lesbian, gay, or bisexual; in 32 states it was legal to fire someone for being transgender. The fact that such harassment and unequal treatment is faced by a significant portion of the planetary science community motivates and requires action over the next decade to create a safe and equitable work environment.

\footnotetext{
${ }^{1} \mathrm{https} / / /$ news.gallup.com/poll/234863/estimate-lgbt-population-rises.aspx

2 https://www.geosociety.org/documents/gsa/about/MbrDemographics.pdf

${ }^{3}$ https://www.supremecourt.gov/opinions/19pdf/17-1618_hfci.pdf
} 
Prior to providing recommendations on how to work towards creating inclusive, supportive, and safe environments across the planetary sciences for members of the LGBTQ+ community, we would like to highlight a few initiatives currently taking place that are effective and that we urge be continued. We recommend the continuation of:

- Employee Resource Groups (ERGs) that have institution-level support such as the NASA Headquarters LGBTQ Pride Alliance ${ }^{4}$ or the Out and Allied ERG at Johnson Space Center. The institutional support of such groups is key to their success, as it removes additional burdens from members of the LGBTQ+ community to self-support their organizations.

- Inclusion of LGBTQ+ events in the full program for conferences and workshops. For example, the listing of the annual LGBTQ+ networking dinner ${ }^{5}$ at the Lunar and Planetary Science Conference (LPSC) each March is included on the official schedule under "Peripheral Meetings and Events". The LPSC also arranges a room for members of the LGBTQ+ community to congregate prior to the event to ensure a safe environment is created for members walking from the conference to the networking dinner location.

- Creating and enforcing codes of conduct and having identifiable members of the community, such as the GSA Respectful, Inclusive, Scientific Event (RISE) initiative ${ }^{6}$, who attendees can easily seek out and report all conduct concerns.

- Encouraging the distribution and wearing of LGBTQ+ NASA Pride pins at planetary science events. These pins have spread throughout the geosciences. Furthermore, the endorsement and wearing of pins by employees of NASA Headquarters is greatly appreciated by members of the LGBTQ+ community and promotes a sense of inclusion in the field.

- The creation of the Equity, Diversity, and Inclusion Working Group (EDIWG) and the representation of each Advisory/Assessment Group (AG) in the building of the steering committee.

It is essential to acknowledge and appreciate actions taken over the last decade, as well as current efforts underway across planetary science to foster the inclusion and safety of the LGBTQ+ community, and encourage the continuation of these efforts, in addition to the recommendations below, over the next decade and beyond.

\subsection{RECOMMENDATIONS}

The following sections include a series of recommendations for actionable items that should be implemented across NASA, planetary science workplaces, professional societies, professional meetings/workshops, and universities to work towards creating inclusive, supportive, and safe environments in planetary sciences for members of the LGBTQ+ community. This is not an exhaustive list of recommendations, but rather a set of achievable actions for the next decade in order to make the community more inclusive.

\subsection{WORKFORCE}

It is critical, both for their work and their personal well-being, that all planetary scientists,

\footnotetext{
${ }^{4}$ https://www.nasa.gov/centers/hq/eodm/employee_resource_groups/pride_alliance

5 https://www.hou.usra.edu/meetings/lpsc2020/events/?date=2020-03-16

6 https://www.geosociety.org/GSA/Events/EventConductCode/GSA/Events/Conduct-intro.aspx
} 
including LGBTQ+ scientists, feel included, supported, and safe in both their workplace and in the broader scientific community. Unfortunately, Cech and Pharm (2017) showed that inequalities experienced by members of the LGBTQ+ workforce are widespread within STEM-related agencies. In addition, Richey et al. (2019) found that LGBTQ+ women and other gender minorities experience more homophobic and transphobic remarks from their peers, compared with their cisgender, straight women colleagues. Richey et al. (2019) also showed that LGBTQ+ women and gender minorities are more likely to be verbally harassed because of their sexual orientation and gender identities, and twice as likely to be physically harassed because of their gender or sex. In addition, Olcott and Downen (2020) ${ }^{7}$ found that $55 \%$ of respondents to a poll of LGBTQ+ geoscientists had done field work or remote research in an area where they did not feel comfortable because of their "identify, expression, or presentation". Furthermore, far fewer graduate students than faculty members felt able to opt out of travel to a location they felt unsafe. To make planetary science workplaces more inclusive, supportive, and safe environments for members of the LGBTQ+ community, we recommend the following implementations (in no particular order).

- Initiate additional NASA-supported funding opportunities, similar to NSF opportunities ${ }^{8}$, to support equity, diversity, inclusion, and accessibility (EDIA) studies. Develop and implement a plan for continued workforce studies over the next decade and beyond.

- Develop clear anti-harassment policies for awarded grants ${ }^{9}$, which include actions if a principal investigator(s) or co-investigator(s) is found to have committed harassment against members of the LGBTQ+ community.

- Request additional demographic information upon submission of NASA-supported proposals. Utilize demographic information to ensure members of the LGBTQ+ community are receiving adequate funding compared with peers who do not identify as LGBTQ+.

- Ensure clearly marked gender-neutral restrooms are available throughout the workplace and that all members of the management team are aware of the bathroom locations.

- Develop ERGs for members of the LGBTQ+ community and allies.

- Ensure any and all paperwork containing sensitive information regarding employees' identity be handled by members of management teams only, and not shared with coworkers, to ensure the privacy of LGBTQ+ employees, allowing them to opt in to visibility.

- Provide benefits to domestic partners of LGBTQ+ employees.

- Provide sufficient health care benefits to LGBTQ+ employees.

- Advertise job announcements in LGBTQ+ spaces and actively recruit, hire, and retain LGBTQ+ employees.

\subsection{PROFESSIONAL SOCIETIES AND MEETINGS}

A recent study of 30 ecology and conservation conferences held since $2009^{10}$ showed almost $40 \%$ of the conferences were held in locations where laws and/or other societal norms discriminated against people of specific sexual orientations or genders. Furthermore, only two of these conferences had explicit information available on their website about how the safety of attendees would be assured. Although we could not find a similar study specific to planetary science conferences, given the similarly global nature of our community one might expect similar results. Since sharing our science with our peers is such a huge part of the success and continuation

\footnotetext{
${ }^{7}$ https://eos.org/features/the-challenges-of-fieldwork-for-lgbtq-geoscientists

e.g., ${ }^{8}$ https://www.nsf.gov/funding/pgm_summ.jsp?pims_id=5383

9 https://www.nsf.gov/news/news_summ.jsp?cntn_id=296610

${ }^{10}$ Tulloch, A.I.T. (2020) Nature Ecol. Evol. http://doi.org/d6nt; 2020
} 
of our field, it is imperative that the LGBTQ+ community is included in decisions made for professional societies and meetings. We recommend the following implementations (in no particular order) to make professional societies and meetings more inclusive, supportive, and safe environments for members of the LGBTQ+ community.

- Include personal gender pronouns on badges either by providing ribbons/stickers or allowing direct printing on the badges (See Strauss et al. white paper for additional inputs).

- Ensure clearly marked gender-neutral restrooms are available. Ensure all staff associated with the conference are aware of their locations. Include locations on meeting information slides/posters.

- Develop a clear anti-harassment policy and code of conduct (see Diniega et al. white paper) for membership in the society/attendance at the meeting. Develop and circulate clear reporting procedures. Strictly adhere to policies and enforce disciplinary actions when necessary.

- Investigate the area prior to meeting selection and ensure it will be safe for members of the LGBTQ+ community $^{11}$ to attend. Remember that many countries still have mandatory jail time, lashings, or even the death penalty for LGBTQ+ persons.

- At any offered "Diversity in Sciences" event or outreach opportunity (e.g. Women in Science or People of Color in Science), include programming specific to LGBTQ+ people in science as well (e.g., panel discussions, workshops, or social media blasts highlighting LGBTQ+ scientists).

- Do not assume the identity of any members of planetary science, especially based on outward appearances, when looking for participants in various diversity events (e.g., womenin-STEM events), as their identity may not match your assumption. Instead, be sure to put open calls for participants out to all members and allow the members to self-identify. Furthermore, after self-identifications are made, do not discriminate against potential participants based on outward appearances or preconceived notions. Lastly, any enacted dress codes must be fluid enough to support outward gender expression.

\subsection{UNIVERSITIES}

To grow the number of LGBTQ+ planetary scientists, we must first support them throughout their education and assist them in pursuing a career in the field. A recent study by Hughes et al. (2018) showed that at least 7\% fewer LGBTQ+ students are retained in STEM fields when compared to their heterosexual peers after completing a college degree (i.e., $71.1 \%$ heterosexual students vs. $63.8 \%$ sexual minority students). We cannot continue to diversify planetary science if we do not first succeed at getting more diverse people into, and also retaining them in, the field. Therefore, we recommend the following implementations (in no particular order) to make planetary science departments more inclusive, supportive, and safe environments for members of the LGBTQ+ community.

- Provide LGBTQ+ undergraduate students planning to attend graduate school with resources so they can find LGBTQ+ friendly universities and departments. In creating such a list, one should be sure to take into account the general area where the university is located, as well as the makeup of the current university and department staff. Obtaining recent insight from current faculty and staff is crucial for developing this list. It would also be beneficial for universities to self-create such lists as resources available to the community.

- Secure at least one restroom in the buildings offering planetary science courses to be

\footnotetext{
${ }^{11}$ https://www.forbes.com/sites/laurabegleybloom/2019/11/25/most-dangerous-places-safest-lgbtq-gaytravelers/\#125769081169
} 
gender-neutral. Ensure students, faculty, and staff alike are aware of these restroom locations. In addition, during field excursions, access to these restrooms should be included in accessibility plans, and accommodations made when they are not available.

- Provide access and support to the department from any LGBTQ+ resource center on site. Have a representative come to any undergraduate and graduate student prospective weekends and orientations to talk to the students about resources available on campus.

- Facilitate safe zones and other LGBTQ+ trainings for the students, faculty, and staff. Encourage trained faculty members to include visible "Safe Zone" signage outside of their offices as a resource to students. Advertise any such trainings on both department websites and in planetary sciences classes.

- Encourage the use of pronoun statements in all official meetings (e.g. as speakers introduce themselves, state, "Hi, my name is and I use pronouns"). Normalize offering one's own pronouns during introductions. Include pronouns in departmental biographies and email signatures. Include pronoun options on all official forms. Allow students to change their pronouns and name in university documents/websites.

- Host speakers and panels to specifically discuss LGBTQ+ issues and inclusion across the department. Advertise any such speakers and panels on both department websites and in planetary sciences classes.

- Host members of the LGBTQ+ planetary sciences community as colloquium speakers. If the colloquium speaker is comfortable with it, during visits (either in person or virtually) set aside time for the colloquium speakers to meet separately with LGBTQ+ members of the department. In addition, if the colloquium speaker is comfortable with it, notify the LGBTQ+ center on campus about the talk and encourage them to advertise it through their network.

- Advertise job announcements in LGBTQ+ spaces and actively recruit, hire, and retain LGBTQ+ employees for faculty and staff positions.

- Develop and distribute department-specific codes of conduct that go above and beyond the ones established by the university to ensure the inclusion, support, and safety of the LGBTQ+ community within the department. Develop protocols for anonymous reporting of discrimination to authorities (department chairs, departmental coordinators), and ensure that these authorities are properly trained in managing such reports through conflict resolution or further disciplinary action.

- Provide both means for assessing LGBTQ+ student experiences and for LGBTQ+ students to provide feedback on issues (e.g., through climate surveys done every few years that incorporate LGBTQ+ issues). Ensure there are ways for students to safely (and when necessary, anonymously) bring up their concerns to the department.

5.0 INCREASE VISIBILITY OF THE LGBTQ+ COMMUNITY ACROSS THE PLANETARY SCIENCES

An important aspect of creating inclusive, supportive, and safe environments for members of the LGBTQ+ community is increasing community visibility across the planetary sciences. Analysis of demographic data provided in Section 2.0 indicates that LGBTQ+ representation has grown in planetary science from $3 \%$ in 1970 to $12 \%$ in the last 5 years. Although some of this growth can be attributed to an increased number of LGBTQ+ scientists in the field, a portion of this number is likely attributed to an increase in visibility across the field and therefore an increased likelihood of disclosure of identity by members of the LGBTQ+ community. Implementation of the following recommendations should lead to further increases in visibility of the LGBTQ+ community across the planetary sciences and therefore a safer, more inclusive and supportive 
environment. Again, these are a few initial steps that can be taken to support the LGBTQ+ community in the next decade; however, we encourage additional actions beyond those listed below to be implemented (see Section 6.0 for more resources and suggestions).

- Organize and advertise LGBTQ+ Networking events at conferences and workshops such as the Queers in Planetary Sciences (QUIPS) networking dinner at LPSC or the various gAyGU events at the annual American Geophysical Union meetings.

- Recognize LGBTQ+ in STEM Day ${ }^{12}$ and support activities and events to showcase this day.

- Supply stickers/ribbons/pins supporting the LGBTQ+ community at conferences and workshops. Examples include regular rainbow pins, queer science flag stickers, rainbow NASA pins ${ }^{13}$, LGBTQ+ in STEM stickers, pronoun stickers/ribbons.

- Create and support webpages for various LGBTQ+ groups such as QUIPS.

- Initiate role model and mentoring opportunities at conferences and workshops, within professional organizations, across university departments and within planetary science research workplaces focused on connecting LGBTQ+ students and early career professionals with more experienced members of the LGBTQ+ community.

- Encourage participation in larger professional organizations and committees such as the National Organization of Gay and Lesbian Scientists and Technical Professionals Inc ${ }^{14}$ and AAS Committee for Sexual-Orientation and Gender Minorities in Astronomy ${ }^{15}$.

\subsection{RESOURCES AND REFERENCES}

We have listed numerous recommendations that should be implemented in the preceding sections. Here, we provide additional resources that can be utilized to 1) implement these recommendations and 2) call attention to other actions that can be taken, above and beyond those described within this white paper, to create inclusive, supportive, and safe environments across the planetary sciences for members of the LGBTQ+ community.

- Ackerman, N., et al. (2018) arXiv:1804.08406 (and resources therein)

- Cech, E.A. and Pham, V. (2017) Soc. Sci, 6(1), 12, doi: 10.3390/socsci6010012

- Gibney et al., (2019) Nature, 571, 16-17: doi: 10.1038/d41586-019-02013-9

- Hendrix et al. (2020) LPSC Abstract \# 2813

- LGBTQ+ Advocacy in STEM (https://lgbtq.asee.org/)

- Marín-Spiotta, E. et al. (2020) Adv. Geosci., 53, 117-127, doi: 10.5194/adgeo-53-117-2020

- Mattheis et al. (2019) Journal of Homosexuality, doi: 10.080/00918369.2019.161032

- Out \& Equal Workplace Advocates Toolkits and Guides (https://outandequal.org/toolkitsguides/)

- Pendergrass, A. et al. (2019) Inclusive Scientific Meetings: Where to Start (https://500womenscientists.org/inclusive-scientific-meetings).

- Richey, C.R. et al. (2019) Bulletin of the AAS, 51(4): https://baas.aas.org/pub/2019i0206

While utilizing the information in this paper, as well as additional input from the resources listed above to inform the next decade of LGBTQ+ inclusion in the planetary sciences, we remind you of the words of George Takei, "We should indeed keep calm in the face of difference, and live our lives in a state of inclusion and wonder at the diversity of humanity".

\footnotetext{
${ }^{12}$ https://prideinstem.org/lgbtstem-day-2019-press-release/

13 https://www.kickstarter.com/projects/spacesciencepridepin/nasa-shaped-pride-pins

14 https://www.noglstp.org/

15 https://aas.org/comms/sgma
} 